\title{
PRODUCCIÓN DE BIOMASA DE BRACHIARIA (Brachiaria brizantha) CV. MARANDÚ EN DOS SISTEMAS SILVOPASTORILES DE PINO (Pinus elliottii) EN CORRIENTES, ARGENTINA
}

\author{
BIOMASS PRODUCTION OF BRACHIARIA (Brachiaria brizantha) CV. \\ MARANDÚ IN TWO SILVOPASTORAL SYSTEMS OF PINUS (Pinus elliottii) \\ IN CORRIENTES, ARGENTINA.
}

\author{
María Valentina Maurig ${ }^{1 *}$ y Aldo C. Bernardis ${ }^{2}$
}

${ }^{1}$ Agencia de Extensión Rural, Instituto Nacional de Tecnología Agropecuaria (INTA), Cnel. Schweizer 824, Esquina, Corrientes CP 3196, Argentina.

2 Facultad de Ciencias Agrarias, Universidad Nacional del Nordeste (UNNE), Sgto. Juan Bautista Cabral 2131, Corrientes CP 3400, Argentina.

* Autor para correspondencia E-mail: maurig.maria@inta.gob.ar

\section{RESUMEN}

Los sistemas silvopastoriles comienzan a cobrar importancia en la región del noreste argentino (NEA), en particular en los bosques implantados a fines de la década de 1990 e inicios del año 2000. El objetivo del presente trabajo fue evaluar el efecto que producen dos técnicas de raleo en un sistema de Pinus elliottii Engelm. sobre la productividad de Brachiaria brizantha Stapf. cv. Marandú. Los tratamientos bajo análisis fueron: T1: Raleo Selectivo (392 árboles ha-1) y T2: Raleo Sistemático (líneas apareadas con callejones de $12 \mathrm{~m} ; 348$ árboles ha-1) sobre los que se evaluó la acumulación de biomasa aérea de la pastura. Se realizó un muestreo estratificado en el cual se definieron tres sectores. Se instalaron 5 jaulas de $1 \mathrm{~m}^{2}$ por cada sector, sumando en total 15 jaulas por cada tratamiento. El material cosechado, después de pesado, se colocó en estufa, obteniéndose el contenido de materia seca ( $\mathrm{kg} \mathrm{MS} \mathrm{ha-1}^{-1}$. Para caracterizar a los sistemas de manejo en función de la luz que atraviesa el dosel arbóreo se estimó radiación fotosintéticamente activa (PAR) en $\mu \mathrm{mol} \mathrm{m}^{-2} \mathrm{~s}^{-1}$. Se realizó un análisis de medidas repetidas empleando modelos mixtos mediante PROC MIXED de SAS. La comparación de medias se realizó con LSMEANS de SAS, mediante el test de Tukey-Kramer a un nivel de confianza de $95 \%$. La acumulación media de biomasa fue superior en el tratamiento con raleo sistemático (T2) $898,3 \mathrm{~kg} \mathrm{MS} \mathrm{ha}^{-1} \mathrm{anono}^{-1}$, mientras que en T1 fue de $750 \mathrm{~kg} \mathrm{MS} \mathrm{ha}^{-1} \mathrm{año}^{-1}$, registrándose diferencias altamente significativas entre ambos tratamientos $(\mathrm{p}<0,0001)$.

Palabras clave: biomasa forrajera, raleo selectivo, raleo sistemático, PAR.

\section{ABSTRACT}

Silvopastoral systems are important in northeastern Argentina (NEA), particularly in forests planted in the late 1990s and at the beginning of 2000. The aim of this study was to evaluate the effect produced by two thinning methods for Pinus elliottii Engelm. on the productivity of Brachiaria brizantha Stapf. cv. Marandú. The treatments under study were: T1: Selective thinning (392 trees $\mathrm{ha}^{-1}$ ) and T2: Systematic thinning (lines paired with alleys of $12 \mathrm{~m}, 348$ trees ha-1). The aboveground biomass accumulation of the pasture was evaluated in both treatments. A stratified sampling was performed and three sectors were defined. Five cages of $1 \mathrm{~m}^{2}$ were installed in each sector, adding a total of 15 cages per treatment. The harvested material was weighed and placed in an oven, and 
then the dry matter content ( $\mathrm{kg} \mathrm{MS} \mathrm{ha-1)}$ was determined. Photosynthetically active radiation (PAR), expressed in $\mu \mathrm{mol} \mathrm{m}^{-2} \mathrm{~s}^{-1}$, was determined in order to characterize the management systems based on the light that crosses the tree canopy. A repeated measures analysis was performed using mixed models through PROC MIXED of SAS. The comparison of means was performed with LSMEANS of SAS, using the Tukey-Kramer test at a confidence level of $95 \%$. The average accumulation of biomass

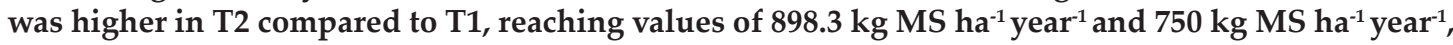
respectively. Significant differences were found between the treatments $(p<0.0001)$.

Key words: forage biomass, selective thinning, systematic thinning, PAR.

\section{INTRODUCCIÓN}

Las provincias que integran la región nordeste de la Argentina (NEA), tienen una superficie de $501.487 \mathrm{~km}^{2}$, con una gran diversidad de suelos, regímenes pluviométricos y vegetación. Abarca tres dominios fitogeográficos (Parque Chaqueño Oriental, Espinal Periestépico y Parque Correntino) cuyo común denominador es la presencia de un estrato arbóreo, de distinta densidad, como componente ecológico regulador de su fisonomía (Chiossone, 2006).

La ganadería es una de las principales actividades económicas del área, que se realiza conjuntamente con el monte natural, y más recientemente como actividad complementaria en los bosques implantados (Pérego, 2002; Chiossone, 2006; Acosta et al., 2009). Esta actividad conjunta se define como Sistema Silvopastoril (SSP). El objetivo general de estos sistemas es maximizar la rentabilidad de un área determinada de tierra mediante la combinación de dos componentes primarios, uno arbóreo y otro herbáceo o forrajero, que es la base de sustentación de la producción secundaria, la bovina (Fassola et al., 2005; Peri et al., 2007; Crespo, 2008).

Los primeros intentos en desarrollar sistemas silvopastoriles en la región del NEA datan de fines de la década de 1970, pero no fueron viables por varias razones, con lo cual el modelo productivo no pudo adaptarse. Sin embargo, estos sistemas comenzaron a difundirse aceleradamente, en particular en los bosques implantados, a fines de la década de 1990 e inicios de la década del 2000 (Esquivel et al., 2004. Lacorte y Esquivel, 2009; Fassola et al., 2009).

En la provincia de Corrientes, los bosques implantados con especies de Pinus y Eucalyptus ocupan una superficie aproximada de 420.000 ha (Elizondo et al., 2015). Sin embargo, aún no se ha desarrollado todo el potencial que tendrían los sistemas silvopastoriles para la región. Según Carvalho et al. (2002) y Clavero y Suárez (2006) existe un conjunto de factores de índole técnico, socioeconómico y sociocultural que ha incidido en que la utilización de los sistemas silvopastoriles no sea la esperada, a pesar de los múltiples bienes $\mathrm{y}$ servicios que ofrecen.

Estos sistemas son capaces de maximizar la rentabilidad de un área dada teniendo en cuenta que la productividad del mismo depende de la competencia que se genera entre sus componentes (Dodd et al., 2005; Devkota et al., 2009; Jose et al., 2009; Udawatta et al., 2014). La presencia de más de un estrato de vegetación presupone conocer y manejar múltiples interacciones ecológicas, que además son dinámicas. La competencia por luz es el principal factor que determina la producción de forrajeras en sistemas silvopastoriles (Carranza y Ledesma, 2009).

Muchos estudios con gramíneas tropicales han mostrado que bajo condiciones de sombra moderada existe evidencia de mantenimiento o incluso aumento de la producción de forraje en comparación con las condiciones de pleno sol (Baruch y Guenni, 2007). En el subtrópico húmedo se ha visto que Axonopus compressus, A. catarinensis y Brachiaria brizantha, aumentan su producción a niveles intermedios de sombra, y se ha medido un aumento de hasta el 70\% de la producción de pastizales de Sorghastrum agrostoides, Andropogon lateralis y Axonopus sp. creciendo bajo $50 \%$ de cobertura (Lacorte y Esquivel, 2009). Sin embargo, cuando los niveles de sombra exceden este nivel de radiación incidente, la producción de forraje se reduce debido a una importante disminución en las tasas fotosintéticas (Devkota et al., 2009; Guenni et al., 2008; Paciullo et al., 2010; Sousa et al. 2007a; b; Gobbi et al., 2009).

Los árboles, al reducir la luminosidad disponible para las plantas que crecen bajo sus copas, tienen influencia sobre los aspectos morfofisiológicos determinantes de la productividad de las mismas (Paciullo et al., 2008). El cambio en la calidad de luz, fundamentalmente la alteración de la relación rojo / rojo lejano, produce cambios morfológicos, como etiolación y aumento de área específica de hojas (Carranza y Ledesma, 2009), cambios en la composición química de las plantas que afectan directamente a la calidad forrajera, cambios en la asignación de biomasa, alterando la relación biomasa raíz / biomasa aérea (Cavagnaro y Trione, 2007; Carranza y Ledesma, 2009) y 
alteración de la producción y calidad de semillas, aspecto fundamental para la persistencia de los pastizales (Castro et al, 2009). Por lo tanto, es importante el conocimiento básico de estas respuestas ecofisiológicas y morfogénicas de las gramíneas bajo condiciones de sombreamiento, ya que pueden determinar un manejo adecuado de estas especies cuando son usadas en sistemas silvopastoriles (Paciullo et al., 2008). En este sentido, los parámetros estructurales del bosque que condicionan la cantidad de luz que llega al sotobosque pueden ser modificados por medio de prácticas silviculturales como los raleos, aumentando el DAP (diámetro a la altura del pecho), disminuyendo la densidad y la cobertura de copas (Peri et al., 2005; Peri, 2007).

De esta forma, el éxito de un sistema silvopastoril va a depender del equilibrio entre los componentes arbóreos y herbáceos, ya que la competencia por recursos para su crecimiento y producción, como la radiación, agua y nutrientes, puede hacer que la sostenibilidad del sistema sea inviable. El objetivo del presente trabajo fue comparar el efecto de dos técnicas de raleo en un sistema silvopastoril de Pinus elliottii Engelm. sobre la productividad de Brachiaria brizantha Stapf. cv. Marandú.

\section{MATERIALES Y MÉTODOS}

El estudio se realizó en el predio Capibara Cué de la Empresa Enrique R. Zeni y Cia. SACIAFel, ubicado sobre la ruta provincial № 30 (Paraje Malvinas), al Norte del Departamento Esquina, Provincia de Corrientes (29은 $30^{\prime \prime}$ S; 59ำ $15^{\prime} 46^{\prime \prime}$ O), Argentina. La temperatura media anual es de $19,5^{\circ} \mathrm{C}$, presentando un marcado descenso de temperatura en el periodo invernal con ocurrencia de algunas heladas. Existe una predominancia de viento norte durante los meses del verano y viento sur durante los meses de invierno, en los demás meses del año predominan vientos con dirección este-nordeste. La precipitación media anual es de $1.275 \mathrm{~mm}$ y su distribución presenta dos máximos, en primavera y en otoño, y un mínimo en invierno. El suelo en el sitio del ensayo es arenoso (Psamacuentes típicos, serie Pampín) (Escobar et al., 1996). El ensayo se instaló sobre una pastura de Brachiaria brizantha cv. Marandú, sembrada en febrero de 2000, previa preparación del terreno en la primavera de 1999 y comienzos del verano del 2000. Para eliminar la biomasa forrajera acumulada y favorecer la plantación y establecimiento de los pinos, fue cortada y enrollada en noviembre de 2000, marzo y diciembre del 2001. La plantación de Pinus elliottii se realizó en octubre de 2000 con un marco de 4 × 2,5 m (1000 árboles ha $\left.{ }^{-1}\right)$ y orientación E-O.
En el año 2004 se realizó un raleo no comercial, quedando 850 árboles ha ${ }^{-1}$ remanentes, los cuales fueron podados hasta $2,5 \mathrm{~m}$ de altura. En septiembre de 2006 se realizó un raleo comercial, quedando así definidos los dos tratamientos bajo análisis:

Tratamiento 1: Sistema silvopastoril con raleo selectivo. El raleo consistió en la corta selectiva de algunos ejemplares de la masa forestal. Se realizó un raleo que consistió en cortar los ejemplares de menor porte (dominados y suprimidos) y dejando los de mayor porte (dominantes y codominantes). La densidad actual de este esquema es de 392 árboles ha-1 ${ }^{-1}$ con una superficie de 15,1 ha.

Tratamiento 2: Sistema silvopastoril con raleo sistemático. La densidad actual es de 348 árboles $\mathrm{ha}^{-1}$. A diferencia del anterior, la técnica de raleo empleada en este tratamiento fue sistemática, eliminándose líneas completas de plantación, descartando ejemplares buenos y malos, quedando así definido un esquema con dos líneas apareadas y callejones entre líneas de 12 metros. La superficie en este esquema silvopastoril es de 15,1 ha.

En el otoño de 2007 se realizó la segunda poda hasta 5,5 $\mathrm{m}$ de altura del fuste, en todos los árboles remanentes.

Se realizó un muestreo estratificado para medir la producción de biomasa forrajera, considerando que la trayectoria del sol varía con el transcurso del día y también con las estaciones del año, afectando así la proyección de las copas de los árboles sobre el estrato herbáceo. Esta sectorización respondió, por lo tanto, a la necesidad de absorber la variabilidad debida a la diferencia en intensidad lumínica que pudiera ser recibida por el estrato herbáceo dentro de cada tratamiento. El día 15 de diciembre de 2009 se comenzó con la instalación de las jaulas y el corte de emparejamiento. Los puntos de muestreo se identificaron en un plano cartográfico y cada uno fue georeferenciado, de manera de ubicarlos en los potreros para el correspondiente muestreo. Para tal fin, se instalaron 5 jaulas de $1 \mathrm{~m}^{2}$ por cada sector, sumando en total 15 jaulas por cada tratamiento. Cada jaula representó una pseudoréplica para el análisis estadístico. La distribución de las jaulas en cada sector se realizó al azar, respetando la metodología que se describe a continuación, para el T1 y T2, respectivamente.

Tratamiento 1: Sector (A): las jaulas se ubicaron en la entrelinea de plantación; Sector (B): las jaulas se ubicaron en las zonas más raleadas ("islas" desprovistas de árboles, producto del raleo selectivo); Sector (C): las jaulas se ubicaron sobre la línea de plantación, entre dos árboles consecutivos.

Tratamiento 2: Sector (A): las jaulas se ubicaron 
en la zona de interfase entre la hilera de árboles y el centro del callejón; Sector (B): las jaulas se ubicaron en el centro del callejón; Sector (C): las jaulas se ubicaron bajo las hileras de plantación.

Las muestras se recolectaron cada 60 días durante el transcurso del año 2010, comenzando en el mes de febrero y culminando en el mes de diciembre. El pasto acumulado en cada jaula se cortó con desmalezadora (motoguadaña), dejando un remanente de $10 \mathrm{~cm}$ sobre la superficie del suelo. Posteriormente, la jaula se colocó de nuevo en el mismo sector, previo corte de emparejamiento (valor cero) para volver a cortar en la próxima fecha. Se evitó colocar 2 jaulas sucesivas en los sitios previamente cosechados, para evitar el efecto del corte previo. El material cosechado, después de pesado, se colocó en estufa hasta peso constante, obteniendo el contenido de materia seca $(\mathrm{kg} \mathrm{MS} \mathrm{ha-1).} \mathrm{Las} \mathrm{muestras} \mathrm{se}$ procesaron en molino tipo Wiley con malla de 1 $\mathrm{mm}$, para ser analizadas en laboratorio.

Se midió la radiación fotosintéticamente activa (PAR) (en $\mu \mathrm{mol} \mathrm{m} \mathrm{m}^{-2} \mathrm{~s}^{-1}$ ) con el objetivo de caracterizar a los tratamientos en cuanto a la proporción de luz que atraviesa el dosel arbóreo. Asumiendo que la radiación transmitida por debajo de los árboles muestra una variabilidad en el tiempo y en el espacio, y que dicha variación puede tener implicancias para la pastura, se realizaron mediciones durante el lapso de un día en invierno y otro día en verano. Las mismas se efectuaron con un ceptómetro de barras integradoras del flujo de fotones de un metro de longitud (AccuPAR ${ }^{\circ}$ model LP-80, Decagon Devices, Inc. Pullman, Washington 99163, USA), en un día diáfano. Las lecturas se tomaron recorriendo cada parcela en transectas con sentido paralelo a la línea de plantación. En este sentido, se respetó la sectorización realizada para el caso de ubicación de las jaulas en cada tratamiento (A, B y C), de modo de integrar todas las situaciones posibles de radiación bajo la canopia. Se tomaron 100 lecturas por cada sector cada una hora, para poder obtener la curva de iluminación durante el día. Para contrastar los valores medidos en el bosque, se tomaron lecturas a cielo abierto (antes de entrar y al salir del bosque). De esta forma se obtuvieron los porcentajes de luz y sombra de cada tratamiento.

Se realizó un análisis estadístico de medidas repetidas empleando modelos mixtos mediante PROC MIXED de SAS (SAS, 2000). El modelo estadístico propuesto fue:

$Y i j k=\mu+J i+T j+P(T) k+\varepsilon i j k$

dónde: Yijk = Es la m-ésima observación en la i-ésima ubicación de la jaula en el j-ésimo tratamiento del k-ésimo período; $\mu=$ Media poblacional; $\mathrm{Ji}=$ Efecto ubicación (i: $1,2,3) ; \mathrm{Tj}$
$=$ Efecto tratamiento (j: 1,2); $\mathrm{P}(\mathrm{T}) \mathrm{k}=$ Efecto período anidado dentro de tratamiento (K: 1, 2, 3, 4, 5, 6); $\varepsilon i j k=$ Error experimental distribuido normalmente con media cero y estructura de covariación.

La comparación de medias se realizó usando LSMEANS de SAS (2000), mediante el test de Tukey- Kramer a un nivel de confianza de 95\%.

\section{RESULTADOS}

Biomasa forrajera. El modelo propuesto para analizar la acumulación de biomasa forrajera señaló diferencias altamente significativas entre ambos tratamientos ( $\mathrm{p}<0,0001)$, y destacó una fuerte interacción con el tiempo $(\mathrm{p}<0,0001)$, no sólo entre períodos dentro de cada tratamiento, sino también entre tratamientos para cada período evaluado. El Tratamiento 2 (SSP con raleo sistemático) fue siempre superior al Tratamiento 1 (SSP con raleo selectivo), demostrado no solo por la acumulación media para el período de ensayo (898,3 vs 750 kg MS ha-1), sino también por los valores medios de biomasa acumulada bimestralmente (Fig. 1).

La mayor diferencia cuantitativa entre ambos tratamientos (265 $\left.\mathrm{kg} \mathrm{MS} \mathrm{ha}^{-1}\right)$ ocurrió al comienzo del ensayo, en la evaluación de febrero, aunque éstas no fueron significativas $(p=0,1820)$. En este período la pastura tuvo su máxima expresión, debido a las altas temperaturas, buena disponibilidad hídrica y radiativa, propias de la época. En cambio, hacia finales del ensayo, la acumulación obtenida en diciembre presentó diferencias altas y significativas $(p=0,0004)$ $(239 \mathrm{~kg} \mathrm{MS} \mathrm{ha-1})$, aunque menores a la del mes de febrero, debido a que normalmente las tasas de crecimiento diario en especies megatérmicas tienen su pico en pleno verano (enero y febrero).

La menor acumulación se registró durante el invierno, período crítico para la producción forrajera. Las bajas temperaturas y los días cortos del período invernal, unido a la baja intensidad de radiación, son las causas fundamentales de la disminución de la productividad de los pastizales. Esto arrojó diferencias significativas entre tratamientos para los meses de junio $(330 \mathrm{y}$ $542 \mathrm{~kg}$ MS ha-1 para el T1 y T2, respectivamente) y agosto (215 y $290 \mathrm{~kg} \mathrm{MS} \mathrm{ha}^{-1}$ para T1 y T2, respectivamente).

Radiación fotosintéticamente activa. En la Tabla 1 se presenta la radiación fotosintéticamente activa (PAR) resultante del promedio de los sectores A, B y C de cada tratamiento, durante un día de invierno (10 de julio) y uno de verano (15 de enero).

Como puede observarse, la PAR recibida por la 


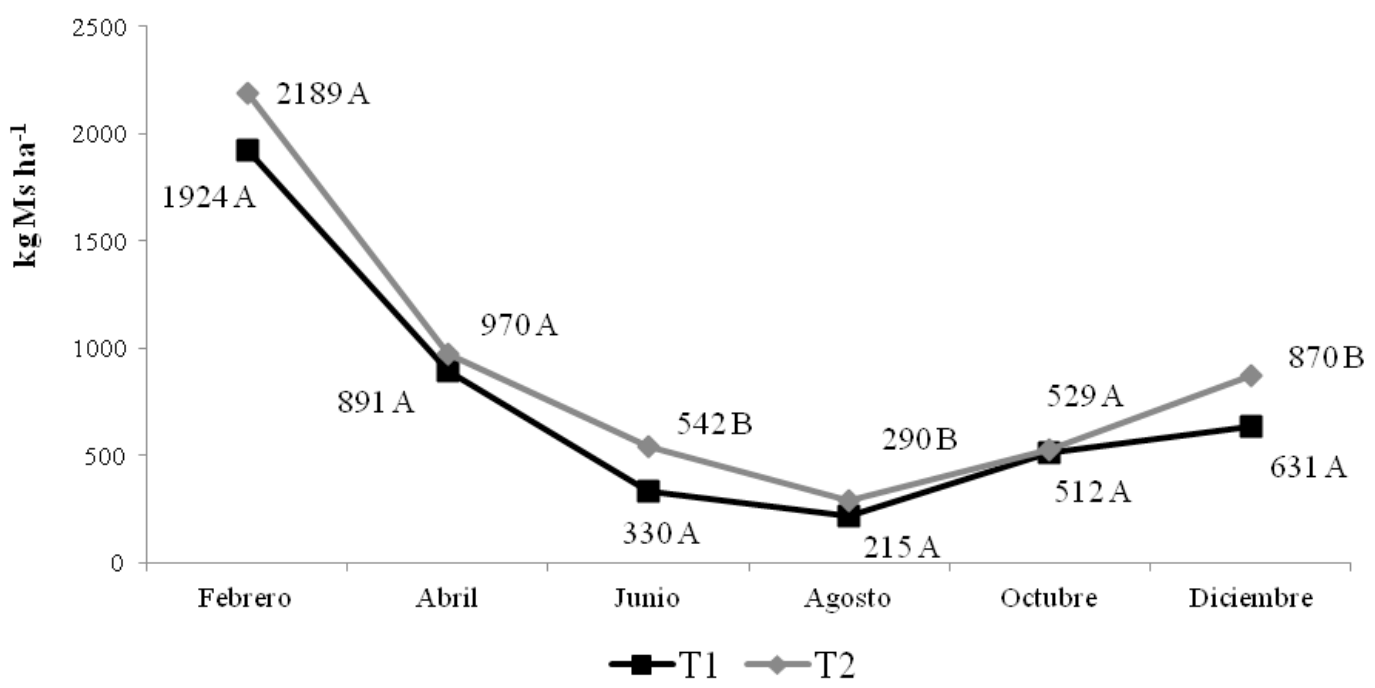

Fig. 1. Valores medios de acumulación de biomasa forrajera (kg MS ha-1) de Brachiaria brizantha $\mathrm{cv}$. Marandú según tratamiento y período de tiempo.

Fig. 1. Mean values of biomass accumulation of forage (DM kg ha-1) of Brachiaria brizantha cv. Marandú for each treatment and period evaluated.

Tabla 1. Valores promedio de radiación fotosintéticamente activa $\left(\mu \mathrm{mol} \mathrm{m} \mathrm{m}^{-2} \mathrm{~s}^{-1}\right)$ recibida por cada sistema (T1 y T2) y valores a cielo abierto (CA).

Table 1. Average values of photosynthetically active radiation $\left(\mu \mathrm{mol} \mathrm{m}^{-2} \mathrm{~s}^{-1}\right)$ received by each system (T1 and T2) and the open sky values (CA).

\begin{tabular}{lcc}
\hline & \multicolumn{2}{c}{ Radiación fotosintéticamente activa PAR promedio } \\
\hline & \multicolumn{2}{c}{$\left(\mu \mathrm{mol} \mathrm{m}^{-2} \mathrm{~s}^{-1}\right)$} \\
Invierno & Verano \\
Cielo abierto (CA) & $728 \mathrm{~B}$ & $1131 \mathrm{~B}$ \\
Tratamiento 1 (T1) & $203 \mathrm{~A}$ & $337 \mathrm{~A}$ \\
Tratamiento 2 (T2) & $236 \mathrm{~A}$ & $475 \mathrm{~A}$ \\
\% PAR T1 & 28 & 30 \\
\% PAR T2 & 33 & 42 \\
\% Sombra T1 & 72 & 70 \\
\% Sombra T2 & 67 & 58 \\
\hline
\end{tabular}

Las diferencias entre tratamientos se analizaron con test de Tukey a un nivel de significancia del 0,05\%.

Porcentajes de sombra, en invierno y verano para T1 y T2, respectivamente. (CA-T1)*100/CA; (CA-T2)*100/CA.

pastura en ambos tratamientos, tanto en invierno como en verano, es muy baja en comparación a la recibida a cielo abierto. También se aprecia que la transmisividad de la radiación en los tratamientos sombreados decrece a medida que decrece el ángulo de elevación solar de verano a invierno. El Tratamiento 1 es el que posee el menor nivel de luminosidad, estando en torno al $30 \%$. Aunque no significativa, la diferencia entre ambos tratamientos se encuentra entre los 5 y 10 puntos porcentuales.

En las siguientes figuras (Figs. 2 a 5) se puede observar la distribución de la luz por sectores bajo el dosel arbóreo y a cielo abierto para ambos tratamientos, en el transcurso de un día de invierno (10 de Julio) y uno de verano (15 de enero), respectivamente. En el caso del T1 se observa que durante el invierno (Fig. 2), la mayor disponibilidad de PAR es recibida en los sectores A (centro de la línea) y B (máxima apertura).

Durante el verano, la transmisividad de la luz resultó superior en el sector $\mathrm{B}$ tal como se observa en la Fig. 3. Para ambos casos (invierno y verano) el sector C (sobre la línea de plantación) es el que 


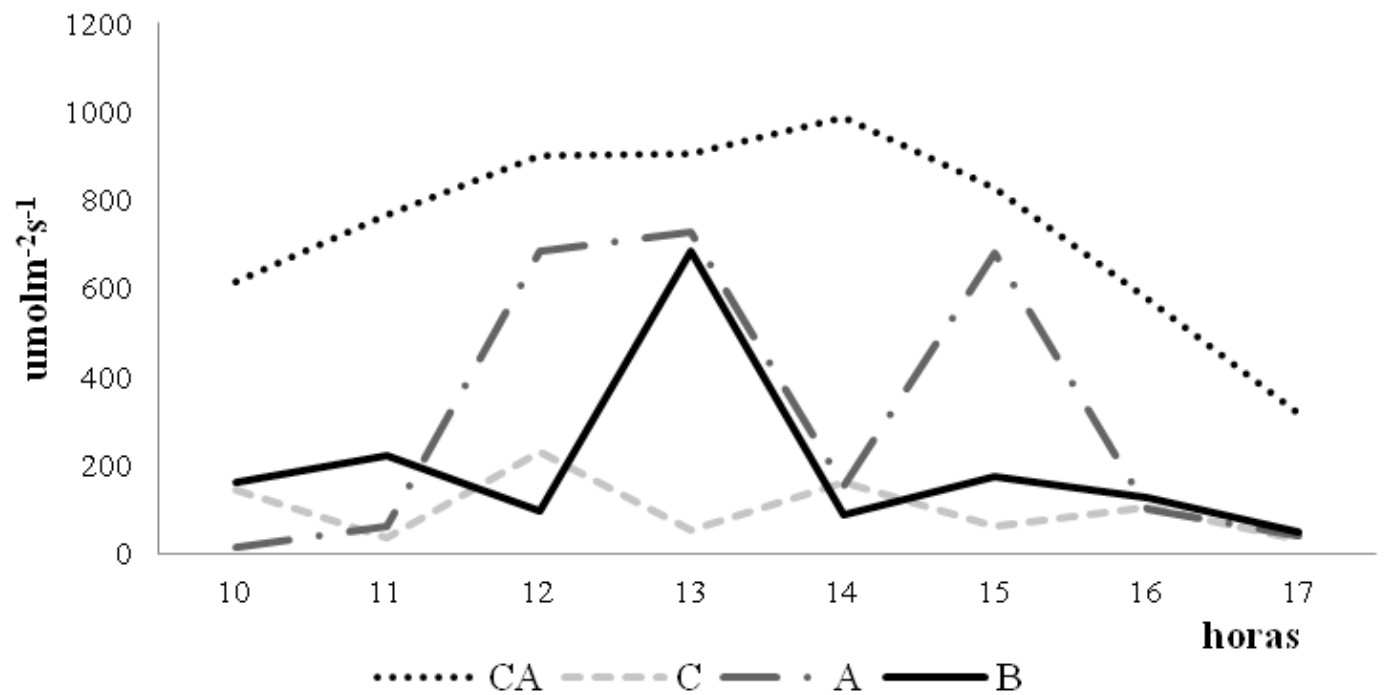

Fig. 2. Distribución diaria de PAR $\left(\mu \mathrm{mol} \mathrm{m} \mathrm{m}^{-2} \mathrm{~s}^{-1}\right)$ por sectores en el T1 en un día de invierno (10 de julio de 2010). CA: cielo abierto; A, B y C: sectores bajo la canopia.

Fig 2. Daily distribution of PAR $\left(\mu \mathrm{mol} \mathrm{m} \mathrm{m}^{-2} \mathrm{~s}^{-1}\right)$ by sectors in T1 on a winter day (July 10, 2010). CA: open sky; A, B and C: sectors under the canopy.

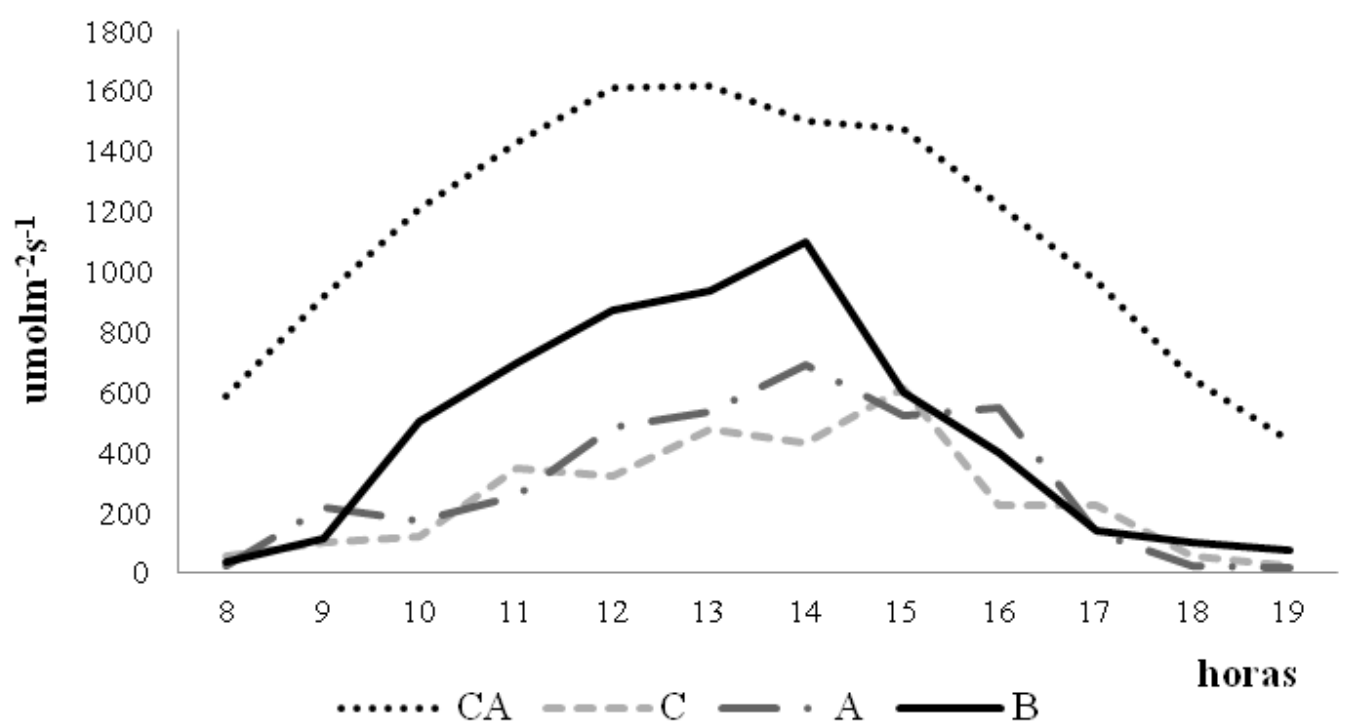

Fig. 3. Distribución diaria de PAR $\left(\mu \mathrm{mol} \mathrm{m}^{-2} \mathrm{~s}^{-1}\right)$ por sectores en el T1 en un día de verano (15 de enero de 2011). CA: cielo abierto; A, B y C: sectores bajo la canopia.

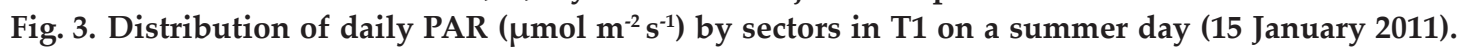
CA: open sky; A, B and C: sectors under the canopy.

recibe la menor incidencia de la luz, no superando en ningún caso el $20 \%$ de la PAR total recibida.

Para el T2 (Figs. 4 y 5) se observa en el sector de máxima apertura (B) una disminución marcada del porcentaje de radiación en los meses de invierno, en contraste con los valores más elevados en el verano. La disponibilidad de PAR bajo la hilera de plantación (sector $\mathrm{C}$ ) también presentó comportamientos diferentes según el momento del año, destacando altos porcentajes en los meses de invierno. Esto se debe a que en los meses invernales el recorrido solar se realiza más hacia el N (en la latitud específica del ensayo), 
situación en la que la proyección de la sombra de las copas se produce sobre los sectores A y B.

Se aprecia que la diferencia en el porcentaje total de PAR recibido en ambos tratamientos es leve y no significativa, pero se observa que la distribución espacial de la luz por sectores difiere entre ambos tratamientos, no solo a lo largo del día sino también durante las diferentes estaciones del año, generando así patrones de luz y sombra heterogéneos en ambos casos.

\section{DISCUSIÓN}

Teniendo en cuenta que la producción promedio de Brachiaria brizantha cv. Marandú a pleno sol medida en el mismo establecimiento es de $8000 \mathrm{~kg} \mathrm{MS} \mathrm{ha-1} \mathrm{año-1} \mathrm{(Dra.} \mathrm{Cristina} \mathrm{Goldfarb,}$ comunicación personal), puede suponerse que las condiciones ambientales prevalentes en el sistema contribuyeron a reducir la producción de la forrajera en ambos tratamientos. Costa et al. (2006), trabajando con Brachiaria brizantha cv. Marandú observaron una disminución del $67,5 \%$ en la producción de la forrajera como consecuencia del sombreamiento, indicando que una disminución de la irradiancia es el principal factor que afecta la producción total de materia seca en las gramíneas, tal como demuestran los resultados del trabajo de Martuscello et al. (2009).

Como se ha mencionado anteriormente, el nivel de sombreo en ambos tratamientos es alto, aún más en el T1, estando en torno al 70\%. Barro et al. (2008) trabajando con genotipos forrajeros invernales como la avena y el trébol; Paciullo et al. (2007) con Brachiaria decumbens, y Andrade et al. (2004) con gramíneas y leguminosas subtropicales, coincidieron con respecto a los efectos negativos sobre la producción y rendimiento de las especies forrajeras con un sombreo igual o superior al $50 \%$. En el caso específico de Brachiaria brizantha, Castro et al. (1999) y Martuscello et al. (2009) recomiendan que el nivel de sombreo no exceda del $60 \%$. Acorde con lo expresado, se observa en este caso que la productividad del recurso forrajero sufrió una disminución del 44\% (4503 kg MS ha- año ${ }^{-1}$ y 33\% (5390 kg MS ha ${ }^{-1} a_{n} o^{-1}$ ) para los tratamientos 1 y 2 , respectivamente, en relación a la producción bajo cielo abierto.

Generalmente, las plantas responden a condiciones medioambientales adversas mediante modificaciones en su tasa de crecimiento y cambios en la movilización de los nutrientes. La productividad de una pastura es un efecto directo de la cantidad de materia seca que una planta puede acumular, lo que depende de la cantidad de puntos de crecimiento que presenta cada planta (Kirchner, 2009). Similar a lo observado en este trabajo, Paciullo et al. (2007), trabajando con Brachiaria decumbens, encontraron que bajo un nivel de $65 \%$ de sombreo la productividad total de la pastura se redujo en un $47 \%$, como consecuencia de una reducción de la misma magnitud en la producción de macollos. Asimismo, las gramíneas cultivadas a la sombra

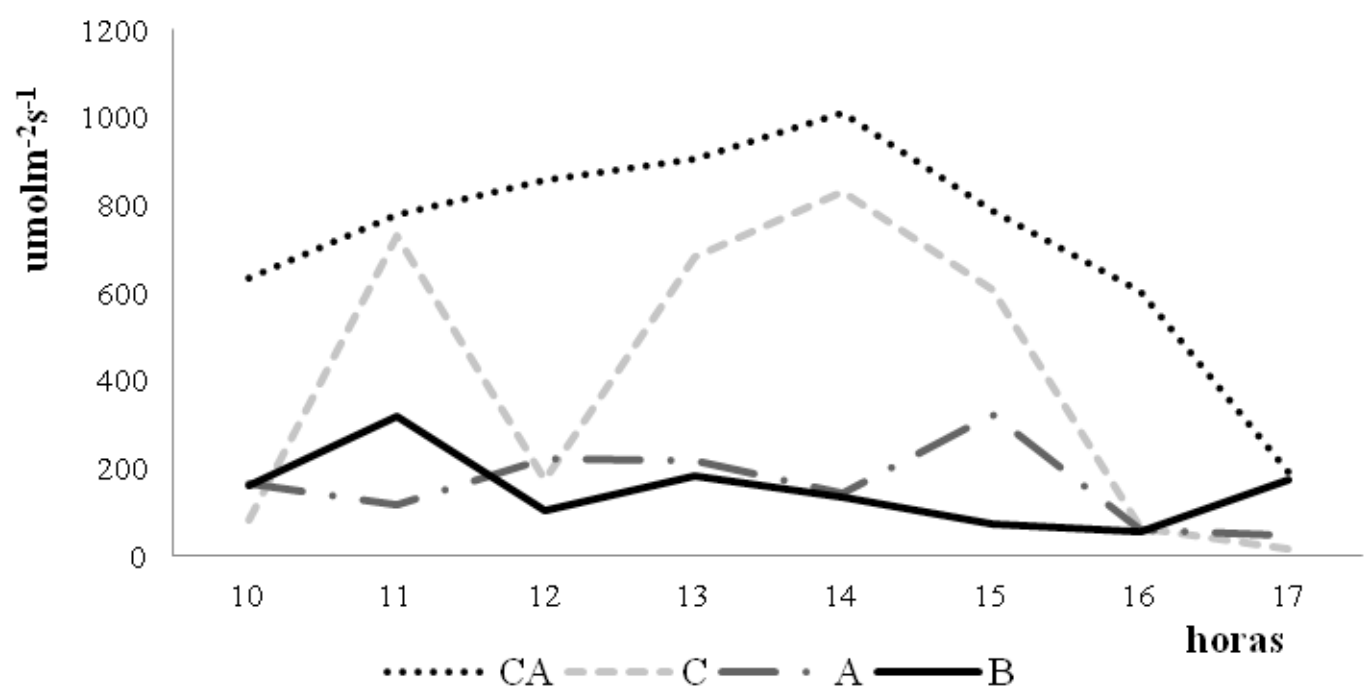

Fig. 4. Distribución diaria de PAR $\left(\mu \mathrm{mol} \mathrm{m} \mathrm{m}^{-2} \mathrm{~s}^{-1}\right)$ por sectores en el T2 en un día de invierno (10 julio 2010). CA: cielo abierto; A, B y C: sectores bajo la canopia.

Fig. 4. Distribution of daily PAR $\left(\mu \mathrm{mol} \mathrm{m} \mathrm{m}^{-2} \mathrm{~s}^{-1}\right)$ by sectors in T2 on a winter day (July 10, 2010). CA: open sky; A, B and C: sectors under the canopy. 


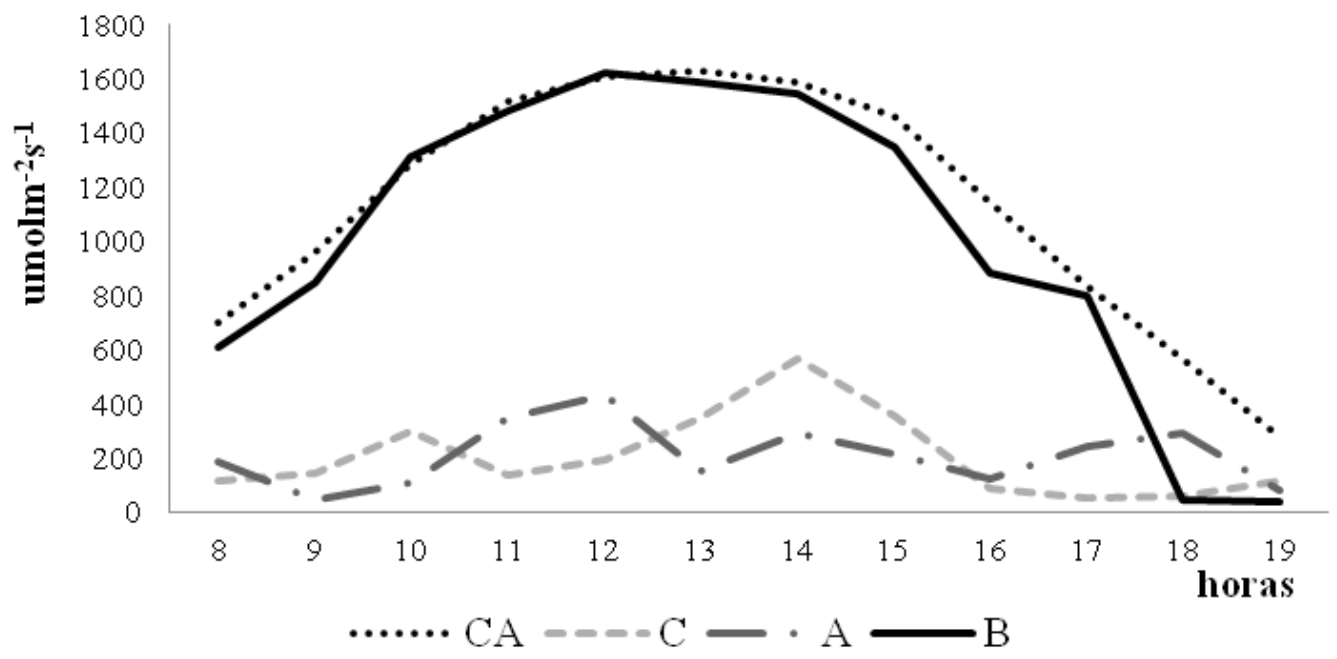

Fig. 5. Distribución diaria de PAR $\left(\mu \mathrm{mol} \mathrm{m} \mathrm{m}^{-2} \mathrm{~s}^{-1}\right)$ por sectores en el T2, para un día de verano (15 enero 2011). CA: cielo abierto; A, B y C: sectores bajo la canopia.

Fig 5. Daily distribution of PAR $\left(\mu \mathrm{mol} \mathrm{m} \mathrm{m}^{-2} \mathrm{~s}^{-1}\right)$ by sectors in T2, for a summer day (15 January 2011 ). CA: open sky; A, B and C: sectors under the canopy.

tienden a ser más suculentas, con menor porcentaje de materia seca, debido al desarrollo más lento de las plantas y la reducida velocidad de pérdida de agua por los tejidos vegetales (Peri et al., 2007).

Por otro lado, la caída de acículas se presenta como un factor deprimente de la productividad de las especies forrajeras en sistemas silvopastoriles con especies de Pinus. Tal como lo indica Kirchner (2009), la competencia por N se hace evidente entre los componentes del sistema. El N utilizado para la descomposición de las acículas, considerando su alta relación C:N, compite con las necesidades nutricionales de las plantas forrajeras, interfiriendo así en su producción de materia seca, sobre todo en el periodo invernal, en donde se produce la mayor acumulación de acículas sobre el estrato herbáceo.

En lo que respecta a las diferencias en acumulación de materia seca entre ambos tratamientos, se puede observar que el T2 resultó superior al T1 a lo largo de cada período evaluado. Aparentemente esta diferencia marcada entre tratamientos pueda ser explicada por la heterogeneidad que presenta la distribución espacial de la luz en cada uno de ellos. Como es de esperar, una sombra uniforme y continua raramente ocurre en este tipo de sistemas, ya que los mismos se caracterizan por tener regímenes fluctuantes con intervalos de luz plena y sombra a lo largo del día (Bahamonde, 2011).

En estos sistemas, la canopia de los árboles absorbe radiación tanto de onda larga como de onda corta del espectro visible (luz roja y azul), ambas efectivas para la fotosíntesis (Jose et al., 2009). En consecuencia, el sotobosque recibe principalmente radiación difusa, compuesta de longitudes de onda media (verde y rojo lejano), siendo estas no eficaces para el proceso fotosintético. Sin embargo, en estos sistemas, la relación rojo/rojo lejano puede variar en gran medida debido a los sunflecks (Ehret et al., 2015). Los sunflecks son fuentes de alta intensidad lumínica que constituyen una fracción sustancial de la luz total disponible para la ganancia de carbono por parte del estrato herbáceo y representan entre un $40-80 \%$ de la luz directa que llega al mismo (Guevara-Escobar et al., 2007). Estas fuentes lumínicas cambian continuamente en su distribución y se dispersan sobre un área amplia dependiendo del arreglo espacial y altura de la forestación, así como de la posición del sol en el cielo, ocasionando alteraciones en el ambiente luminoso y en la actividad fotosintética del estrato herbáceo (Guevara-Escobar et al., 2012).

Según Oker-Blom et al. (1989) y Caballé et al. (2011), la eficiencia de conversión entre la radiación que es interceptada por el estrato herbáceo y su consecuente fotosíntesis depende de la distribución, tanto temporal como espacial, de la irradiancia sobre la pastura. La misma es el resultado integrado de la distribución de la radiación incidente durante un período específico y de la estructura que posee la canopia. Este concepto queda bien demostrado en las Figs. 2 a 5 , donde la diferencia en distribución del dosel 
arbóreo entre ambos tratamientos, sumado a las variaciones típicas de luz estacional y diaria, ocasionan diferentes patrones de luz y sombra. Como puede observarse en la Fig. 1, la mayor diferencia cuantitativa de producción entre tratamientos se registra en el periodo estival, en coincidencia con una mayor incidencia de la luz, y mayor duración, sobre el callejón (sector B) en el Tratamiento 2. En este contexto, Montero (2008) analizó la influencia del esquema de plantación sobre la cantidad de luz que era interceptada por los árboles, encontrando diferencias significativas $(p=0,02)$ entre aquellos esquemas con espaciamientos más estrechos y aquellos más amplios, dando como resultado una mayor intercepción de luz por parte de las copas de los árboles en los esquemas menos espaciados. En coincidencia, Monteith el al. (1991) y Burner y Belesky (2008) aseguran que, en términos microclimáticos, la principal ventaja que poseen los sistemas con callejones es el de permitir mayor absorción de luz por parte del estrato herbáceo a lo largo del año, y como consecuencia una mayor acumulación de biomasa.

Por lo tanto, sería recomendado que, antes de establecer un nuevo sistema silvopastoril, se tomen decisiones en base a la estructura de plantación (densidad, configuración, orientación) y al manejo de los árboles (podas y raleos) con el fin de minimizar las interacciones competitivas entre los componentes del sistema, de modo que el mismo sea sustentable en el tiempo. (Montero et al., 2008).

\section{CONCLUSIÓN}

De acuerdo a los resultados obtenidos se concluye que existen diferencias altamente significativas $(<0,0001)$ en la acumulación de biomasa de Brachiaria brizantha cv. Marandú entre ambos tratamientos. Este trabajo demostró que las interacciones entre los distintos componentes de un sistema silvopastoril son dinámicas y complejas, con relaciones activas que generan cambios entre sus componentes y que, dentro de estas interacciones, la restricción luminosa apareció como uno de los factores más limitantes en la productividad de la pastura. Si bien se aportó conocimiento sobre el efecto que la distribución en el espacio del componente arbóreo produjo sobre el estrato herbáceo, mostrando que el T2 produjo un 16,5\% más (887 kg MSha ${ }^{-1}$ ) que el T1, se debe tener en cuenta que este trabajo corresponde a un período establecido de tiempo, con condiciones ambientales determinadas para ese período, en el que se evaluaron relaciones parciales entre algunos componentes del sistema.

\section{RECONOCIMIENTOS}

A la empresa Enrique Zeni y Cia. SACIAFel por facilitar sus instalaciones y al INTA (Instituto de Tecnología Agropecuaria) por financiar el proyecto. Especial agradecimiento a la Dra. Cristina Goldfarb por ser guía en los ensayos y a la Lic. Laura Giménez por su apoyo en el análisis estadístico.

\section{LITERATURA CITADA}

Acosta, F., L. Giménez, C. Richieri, y M. Calvi. 2009. Zonas agroeconómicas homogéneas Corrientes: descripción ambiental, socioeconómica y productiva. Estudios Socioeconómicos de la Sustentabilidad de los Sistemas de Producción y Recursos Naturales $\mathrm{N}^{\circ}$ 8. ISSN 1851-6955. Centro Regional INTA Corrientes, Corrientes, Argentina.

Andrade, C.M.S. de, J.F. Valentim, J. da Costa Carneiro, e F.A. Vaz. 2004. Crescimento de gramineas e leguminosas forrageiras tropicais sob sombreamento. Pesquisa Agropecuária Brasileira 39(3):263-270.

Bahamonde, H. 2011. Efecto de variables ambientales sobre la productividad primaria neta aérea y la concentración de proteína bruta de gramíneas en sistemas silvopastoriles de ñire (Nothofagus antarctica): creación de un modelo de simulación. Tesis de posgrado. Escuela para Graduados Ing. Agr. Alberto Soriano. Facultad de Ciencias Agrarias. Universidad de Buenos Aires (UBA), Buenos Aires, Argentina.

Barro, R.S., J.C.D. Saibro, R.B.D. Medeiros, J.L.S. Silva, e A.C. Varella. 2008. Rendimento de forragem e valor nutritivo de forrageiras de estação fria, submetidas a sombreamento por Pinus elliottii e ao sol pleno. Revista Brasileira de Zootecnia 37(10):1721-1727.

Baruch, Z., y O. Guenni. 2007. Irradiance and defoliation effects in three species of the forage grass Brachiaria. Tropical Grasslands 41:269-276.

Burner, D.M., and D.P. Belesky. 2008. Relative effects of irrigation and intense shade on productivity of alley-cropped tall fescue herbage. Agroforestry Systems 73(2):127-139.

Caballé, G., M.E. Fernández, J. Gyenge, A. Aparicio, and T. Schlichter. 2011. Modeling leaf maximum net photosynthetic rate of Festuca pallescens, the dominant perennial grass of Patagonian pine-based silvopastoral systems. Agroforestry Systems 83(1):13-24. 
Carvalho, M.M., M.J. Alvim, e D.F. Xavier. 2002. Estabelecimento de sistemas silvipastoris: ênfase em áreas montanhosas e solos de baixa fertilidade. Circular Técnica № 68.11 p. EMBRAPA, Juiz de Fora, MG, Brasil.

Carranza, C.A., y M. Ledesma. 2009. Bases para el manejo de sistemas silvopastoriles. p. 1-9. En Anales XIII Congreso Forestal Mundial. FAO. 18-23 octubre de 2009. Buenos Aires, Argentina. Disponible en https:// ecaths1.s3.amazonaws.com/forrajicultura/ ManejoSistemasSilvopastoriles.pdf (Consulta 20 de febrero 2015).

Castro, C.D., R. Garcia, M.M. Carvalho, e L. Couto. 1999. Produção forrageira de gramíneas cultivadas sob luminosidade reduzida. Revista Brasileira de Zootecnia 28:919-927.

Castro, C.R.T., D.S.C. Paciullo, C.A.M Gomide, V.B.F. Araujo, V.F. Souza, y F.S. Sobrino. 2009. Producción y calidad fisiológica de semillas y aparición de plantas nuevas de Brachiaria decumbens Stapf cv. Basilisk en una pastura en un sistema silvopastoril. p. 164-170. En I Congreso Nacional de Sistemas Silvopastoriles. Instituto Nacional de Tecnología Agropecuaria (INTA). 14-16 de mayo de 2009. Ediciones INTA. En actas, CD. Posadas, Misiones. Argentina.

Cavagnaro J.B., y S.O. Trione. 2007. Physiological and biochemical responses to shade of Trichloris crinita, a forage grass from the arid zone of Argentina. Journal of Arid Environments 68:337-347.

Chiossone, G. 2006. Sistemas de producción ganaderos del noreste argentino. Situación actual y propuestas tecnológicas para mejorar su productividad. p. 120-137. X Seminario de pastos y forrajes. Universidad del Zulia- Luz, FCV Maracaibo, Venezuela. 20-22 de abril de 2006. Disponible en https://www.produccionanimal.com.ar/informacion-tecnica/origenesevolucion-y-estadisticas-de-la-ganaderia/65Guillermo-Chiossone.pdf (Consulta 22 de febrero de 2015).

Clavero T., y J. Suárez. 2006. Limitaciones en la adopción de los sistemas silvopastoriles en Latinoamérica. Pastos y Forrajes 29(3):307-317.

Costa N. de L., C.R. Townsend, J.A. Magalhães, R.G. de A Pereira, D.M.M.R. Azevedo. 2006. Comportamento forrageiro da Brachiaria brizantha cv. Marandu em sistema silvipastoril na Amazônia Brasileira. Pasturas Tropicales 28(3):22-26.

Crespo, G. 2008. Importancia de los sistemas silvopastoriles para mantener y restaurar la fertilidad del suelo en las regiones tropicales. Revista Cubana de Ciencia Agrícola 42(4):329335.
Devkota, N.R., P.D. Kemp, J. Hodgson, I. Valentine, and I.K.D. Jaya. 2009. Relationship between tree canopy height and the production of pasture species in a silvopastoral system based on alder trees. Agroforestry Systems 76:363-374.

Dodd, M.B., A.W. McGowan, I.L. Power, and B.S. Thorrold. 2005. Effects of variation in shade level, shade duration and light quality on perennial pastures. New Zealand Journal of Agricultural Research 48:531-543.

Ehret, M., R. Graß, and M. Wachendorf. 2015. The effect of shade and shade material on white clover/perennial ryegrass mixtures for temperate agroforestry systems. Agroforestry Systems 89(3):557-570.

Elizondo, M.H., J.M. Mestres, J.E. Sens Hourcade, J.C. Moreno, E. Wabo, y A.M. Álvarez. 2015. Actualización del inventario de plantaciones forestales de la provincia de Corrientes. Informe final. En línea. Consejo Federal de Inversiones. Gobierno de la provincia de Corrientes, Argentina. Disponible en http:// recursosforestales.corrientes.gob.ar/assets/ articulo_adjutos/1777/original/Informe_ Final_Inventario_2015.pdf. 14507927766. (Consulta 26 de enero 2015)

Escobar, E.H., H.D Ligier, R. Melgar, H. Matteio, y O. Vallejos. 1996. Mapa de Suelos de la Provincia de Corrientes 1:500.000. 329 p. Instituto Nacional de Tecnología Agropecuaria. Área de Producción Vegetal y Recursos Naturales. EEA El Sombrerito, El Sombrero, Corrientes, Argentina.

Esquivel, J., H.E. Fassola, S.M. Lacorte, L. Colcombet, E. Crechi, A.N. Pachas. 2004. Sistemas Silvopastoriles. Una sólida alternativa de sustentabilidad social, económica y ambiental. p. 11. En Actas XI Jornadas Técnicas Forestales y Ambientales. 07 al 09 de octubre de 2004. Eldorado, Misiones, Argentina. Disponible en CD. Facultad de Ciencias Forestales-UNaM y EEA Montecarlo-INTA.

Fassola, H. E., A.N. Pachas y S.M. Lacorte. 2005. Estimación de la radiación fotosintéticamente activa (PAR) y niveles de sombra bajo dosel de Pinus taeda en la Provincia de Misiones y NE de Corrientes. En III Congreso Forestal Argentino y Latinoamericano. 06 al 09 de septiembre de 2005. Corrientes, Argentina. Publicación disponible en CD. AFOA y Gobierno de Corrientes, Argentina. 
Fassola H.E., S.M. Lacorte, A.N. Pachas, C. Goldfarb, J. Esquivel, L. Colcombet, et al. 2009. Los sistemas silvopastoriles en la región subtropical del NE argentino. p. 1-8. En Anales XIII Congreso Forestal Mundial. FAO. 18-23 de octubre 2004. Buenos Aires, Argentina. Disponible en http://inta.gob.ar/ si-tes/default/files/script-tmp-inta-sistemassilvo-ne-argentino.pdf. (Consulta 20 de febrero de 2015)

Gobbi K.F., R. Garcia, e A.F. Garcez Neto. 2009. Características morfológicas, estruturais e produtividade do capim-braquiária e do amendoim forrageiro submetidos ao sombreamento. Revista Brasileira de Zootecnia 38(9):1645-1654.

Guenni, O., S. Seiter, and R. Figueroa. 2008. Growth responses of three Brachiaria species to light intensity and nitrogen supply. Tropical Grasslands 42:75-87.

Guevara-Escobar, A., P.D. Kemp, A.D. Mackay, and J. Hodgson. 2007. Pasture production and composition under poplar in a hill environment in New Zealand. Agroforestry Systems 69(3):199-213.

Guevara-Escobar, A., M. Cervantes-Jiménez, H. Suzán-Azpiri, E. González-Sosa, e I. Saavedra. 2012. Producción de pasto Rhodes en una plantación de eucalipto. Agrociencia 46(2):175-188.

Jose S., E.J. Holzmueller, H.E. Garrett, and A.R. Gillespie. 2009. Tree crop interactions in temperate agroforestry. p. 57-73. In H.E. Garrett (ed.) North American agroforestry: an integrated science and practice. 2nd ed. American Society for Agronomy, Madison, Wisconsin, USA.

Kirchner, R. 2009. Desempenho de forrageiras anuais de inverno sob distintos níveis de irradiância. Tese (Mestrado). Universidade Tecnológica Federal do Paraná. Pato Branco, Brasil. 93 p. Disponible en www.utfpr.edu.br (Consulta 26 de febrero 2015).

Lacorte, S.M., y J.I. Esquivel. 2009. Sistemas silvopastoriles en la Mesopotamia Argentina. Reseña del conocimiento, desarrollo y grado de adopción. p. 70-82. En Actas 1ํㅡㄹ Congreso Nacional de SSP. Posadas, Misiones. Argentina. 14 al 16 de mayo de 2009. Ediciones INTA, Posadas, Misiones, Argentina.

Martuscello, J.A., L. Jank, M.G. Neto, V.A. Laura e D.N. 2009. Produção de gramíneas do gênero Brachiaria sob níveis de sombreamento. Revista Brasileira de Zootecnia 38:1183-1190.

Monteith, J.L., C.K. Ong, and J.E. Corlett, 1991. Microclimatic interactions in agroforestry systems. Forest Ecology and Management 45(1-4):31-44.
Montero, M.J., G. Moreno, and M. Bertomeu. 2008. Light distribution in scattered-trees open woodlands in Western Spain. Agroforestry System 73:233-244.

Oker-Blom, P., T. Pukkala, and T. Kuuluvainen. 1989. Relationship between radiation interception and photosynthesis in forest canopies: effect of stand structure and latitude. Ecological Modelling 49:73-87.

Paciullo, D.S.C., C.A.B de Carvalho, L.J.M. Aroeira, M.J.F Morenz, F.C.F Lopes e R.O.P. Rossiello. 2007. Morfofisiologia e valor nutritivo do capim-braquiária sob sombreamento natural e a sol pleno. Pesquisa Agropecuária Brasileira 42(4):573-579.

Paciullo, D.S.C., N.R. Campos, C.M.A. Gomide, C.D. Castro, R.C. Tavela, e R.O. Rossiello. 2008. Crescimento de capim-braquiária influenciado pelo grau de sombreamento e pela estação do ano. Pesquisa Agropecuária Brasileira 43(7):917-923.

Paciullo, D.S.C., C.R.T. Castro, C.A.M. Gomide, P.B. Fernandes, W.S.D. Rocha, M.D. Müller, et al. 2010. Soil bulk density and biomass partitioning of Brachiaria decumbens in a silvopastoral system. Scientia Agricola 67:401-407.

Pérego, J.L. 2002. Sistemas silvopastoriles en el centro sur de la provincia de Corrientes. En la XIX Reunión del Grupo Técnico en Forrajeras del Cono Sur - Zona Campos. Publicado en Reunión del grupo técnico en forrajeras del cono sur zona campos, XIX. Mercedes, Corrientes, Argentina. Memorias INTA EEA Mercedes. Disponible en www.produccionanimal.com.ar (Consulta octubre 2002).

Peri, P., M.V.Sturzenbaum, L. Monelos, E. Livraghi, R. Christiansen, A. Moreto, et al. 2005. Productividad de sistemas silvopastoriles en bosques nativos de ñire (Nothofagus antarctica) de Patagonia Austral. En Actas III Congreso Forestal Argentino y Latinoamericano. 06 al 09 de septiembre de 2005. Corrientes. Argentina. Comisión Nuevas Tendencias Forestales. Volumen 10. AFOA y Gobierno de Corrientes, Argentina.

Peri, P.L., R.J. Lucas, and D.J. Moot. 2007. Dry matter production, morphology and nutritive value of Dactylis glomerata growing under different light regimes. Agroforestry Systems 70(1):63-79.

SAS. 2000. Statistical Analysis Systems. Version 8. SAS Institute, Cary, North Carolina, USA. 
Sousa, L.F., R.M. Maurício, E. de O.S. Saliba, e G.R. Moreira. 2007a. Produtividade e valor nutritivo da Brachiaria brizantha cv. Marandu em um sistema silvipastoril. Arq. Bras. Med. Vet. Zootec. 56:658-664.

Sousa L.F., R.M. Maurício, e L.C. Goncalves. 2007b. Produtividade e valor nutritivo da Brachiaria brizantha cv. Marandu em um sistema silvipastoril. Arq. Bras. Med. Vet. Zootec. 59:1029-1037.

Udawatta R.P., P.P. Motavalli, S. Jose, and K.A. Nelson. 2014. Temporal and spatial differences in crop yields of a mature silver maple alley cropping system. Agronomy Journal 106:407-415. 